\title{
A SUFFICIENT CONDITION FOR CONJUGACY OF METRIC AUTOMORPHISMS
}

\author{
BY \\ N. F. G. MARTIN( $\left.{ }^{1}\right)$
}

1. Introduction. In this paper we take an idea of Rota's [8] and define a "universal" metric automorphism on a product space such that if $T$ is any metric automorphism of one of the factor spaces there is an invariant subalgebra of the domain of the universal automorphism such that the universal automorphism restricted to this subalgebra is conjugate to $T$. Those subalgebras in the domain of the universal automorphism which are associated with some metric automorphism on the factor space are characterized. Using these subalgebras it is possible to define a function $h$ on the cartesian product of the group $\mathscr{G}$ of all metric automorphisms of a given measure algebra with itself to the real numbers such that if $T$ and $S$ are conjugate automorphisms then $h(T, S)=0$. Furthermore it is proven that if $h(T, S)=0$ then $T$ and $S$ are "approximately" conjugate in the sense that for every $\varepsilon>0$ there exists an automorphism $R$ and a $T$ invariant subalgebra $\mathscr{B}$ such that $T=R^{-1} S R$ for all sets in $\mathscr{B}$ and if $E$ is a measurable set not in $\mathscr{B}$, then $\mu\left(T E \triangle R^{-1} S R E\right)<\varepsilon$ (Theorem 4.19). As a corollary to this result we obtain that if $h(T, S)=0$ and the conjugacy class of $S$ is closed in the uniform topology in $\mathscr{G}$ then $T$ and $S$ are conjugate.

2. Notation and definitions. Throughout, $(X, \mathscr{M}, \mu)$ will denote a totally finite measure space with $\mu(X)=1$, i.e., a probability space. We shall be concerned with functions defined on the measure algebra of $\mathscr{M}$, that is the measurable sets mod sets of measure zero, and set equalities will be understood to hold almost everywhere. A metric automorphism of $\mathscr{M}$ is a boolean $\sigma$-isomorphism of the measure algebra onto itself which preserves the measure. The collection of all metric automorphisms of $\mathscr{M}$ will be denoted by $\mathscr{G}$. If $T_{1}$ and $T_{2}$ are metric automorphisms of measure algebras $\mathscr{M}_{1}$ and $\mathscr{M}_{2}$ respectively then $T_{1}$ and $T_{2}$ are conjugate (or isomorphic) if there exists a measure preserving boolean $\sigma$-isomorphism $R$ on $\mathscr{M}_{1}$ onto $\mathscr{M}_{2}$ such that $T_{2} R=R T_{1}$. If $T$ and $S$ are in $\mathscr{G}$, then $T$ and $S$ are conjugate if there exists $R \in \mathscr{G}$ such that $T=R^{-1} S R$.

If $\mathscr{C}$ is a sub $\sigma$-algebra of $\mathscr{M}$, the conditional probability of a set $A \in \mathscr{M}$ given $\mathscr{C}$ will be denoted by $\mu(A \mid \mathscr{C})$. For properties of the condition probability see [5] or [2]. If $\xi$ is a finite measurable partition of $X$ into sets $E_{1}, E_{2}, \ldots, E_{n}$, the entropy of $\xi, H(\xi)$, is defined by $H(\xi)=-\sum_{j=1}^{n} \mu\left(E_{j}\right) \log \mu\left(E_{j}\right)$; the relative

Received by the editors September 13, 1965.

(1) Research supported in part by ARO Grant 662. 
entropy of $\xi$ given $\mathscr{C}, H(\xi \mid \mathscr{C})$, is defined by $H(\xi \mid \mathscr{C})=-\sum_{j=1}^{n} \mu\left(E_{j} \mid \mathscr{C}\right) \log \mu\left(E_{j} \mid \mathscr{C}\right)$; and the mean conditional entropy of $\xi$ given $\mathscr{C}, \bar{H}(\xi \mid \mathscr{C})$, is defined by $\bar{H}(\xi \mid \mathscr{C})=$ $\int_{X} H(\xi \mid \mathscr{C}) d \mu$. It is true that $\bar{H}(\xi \mid \mathscr{C})=-\int_{X} \sum_{j=1}^{n} \chi_{E_{j}} \log \mu\left(E_{j} \mid \mathscr{C}\right) d \mu$; if $\mathscr{C}_{1} \subset \mathscr{C}_{2}$ then $\bar{H}\left(\xi \mid \mathscr{C}_{1}\right) \geqq \bar{H}\left(\xi \mid \mathscr{C}_{2}\right)$; and if $\left\{\mathscr{C}_{n}\right\}$ is an increasing sequence of subalgebras of $\mathscr{M}$ and $\mathscr{C}=\bigvee_{n=1}^{\infty} \mathscr{C}_{n}$, then $\lim _{n} \bar{H}\left(\xi \mid \mathscr{C}_{n}\right)=\bar{H}(\xi \mid \mathscr{C})$, a.e. For proofs of these statements see for example [4], [2] or [1]. If $\left\{\mathscr{C}_{a}: a \in A\right\}$ is a collection of subalgebras of $\mathscr{M}$ then $\bigvee_{a \in A} \mathscr{C}_{a}$ will denote the boolean $\sigma$-algebra generated by the sets in $\bigcup_{a \in A} \mathscr{C}_{a}$. If $T$ is a metric automorphism of $\mathscr{M}$ then the entropy of $T, h(T)$, is defined by

$$
h(T)=\sup \left\{\lim _{n \rightarrow \infty} \frac{1}{n} H\left(\bigvee_{j=0}^{n-1} T^{j} \xi\right): \xi \text { finite partition of } X\right\}
$$

One may consult Rohlin's survey article [7] for properties of $h(T)$ and its use in ergodic theory, or Jacob's lecture notes [4].

3. A universal metric automorphism. Let $Z$ denote the integers and $\mathscr{P}$ the collection of all subsets of $Z$. The $\sigma$-algebra $\mathscr{P} \times \mathscr{M}$ will be denoted by $\mathscr{C}$ and $u$ will denote the function on $Z \times X$ defined by $u(k, x)=(k+1, x)$.

3.1. LEMMA. There exists a finitely additive measure $\lambda$ on $\mathscr{C}$ such that $\lambda(Z \times X)=1$ and for every $E \in \mathscr{C}, \lambda\left(u^{-1}(E)\right)=\lambda(E)$.

Proof. Let $V$ denote the real linear space of all bounded real functions on the integers with sup norm and usual definitions of addition and scalar multiplication. By use of the Hahn-Banach theorem (see [9, p. 112]) there exists a positive linear functional $L$ on $V$ such that $L(x)=\lim _{n \rightarrow \infty}(1 / n) \sum_{j=0}^{n-1} x(j)$, if this limit exists, and if one defines $T_{n}$ on $V$, by $\left(T_{n} x\right)(j)=x(j+n)$ for any $n \in Z$, then $L\left(T_{n} x\right)=L(x)$.

Now, for $E \in \mathscr{C}, k \in Z$, let $E_{k}$ denote the set $\{x:(k, x) \in E\}$. Then $\left\{\mu\left(E_{k}\right): k \in Z\right\}$ $\in V$ and we define $\lambda(E)$ to be $L\left\{\mu\left(E_{k}\right): k \in Z\right\}$. Let $E^{1}$ and $E^{2}$ be disjoint sets from $\mathscr{C}$ and denote their related sequences $\left\{\mu\left(E_{k}^{1}\right): k \in Z\right\}$ and $\left\{\mu\left(E_{k}^{2}\right): k \in Z\right\}$ by $x_{1}$ and $x_{2}$ respectively. Since $\left(E^{1} \cap E^{2}\right)_{j}=E_{j}^{1} \cap E_{j}^{2}$, the disjointness of $E^{1}$ and $E^{2}$ implies that $E_{j}^{1} \cap E_{j}^{2}=\varnothing$ and hence $\mu\left(E_{j}^{1} \cup E_{j}^{2}\right)=\mu\left(E_{j}^{1}\right)+\mu\left(E_{j}^{2}\right)$ so that

$$
x_{1}+x_{2}=\left\{\mu\left(E_{j}^{1}\right)+\mu\left(E_{j}^{2}\right): j \in Z\right\}=\left\{\mu\left(E_{j}^{1} \cup E_{j}^{2}\right): j \in Z\right\} .
$$

Hence,

$$
\lambda\left(E_{1} \cup E_{2}\right)=L\left(x_{1}+x_{2}\right)=L\left(x_{1}\right)+L\left(x_{2}\right)=\lambda\left(E_{1}\right)+\lambda\left(E_{2}\right) .
$$

The fact that $\lambda \geqq 0$ and $\lambda(\varnothing)=0$ follows from the fact that $L$ is a positive linear functional.

It is clear that $\lambda$ may not be countably additive, since each of the sets $\{(j, x): x \in X\}$ has $\lambda$-measure zero and $Z \times X=\bigcup_{j \in Z}\{(j, x): x \in X\}$ has $\lambda$-measure one. 
3.2. Definition. For each set $E \in \mathscr{C}$, define the set function $U$ by

$$
U(E)=\{(k, x): u(k, x) \in E\} .
$$

Since $U$ is the inverse image of sets under a point function, $U$ is a $\sigma$-homomorphism on $\mathscr{C}$, and since $u$ is measurable this $U$ maps $\mathscr{C}$ into $\mathscr{C}$.

\subsection{LEMMA. The homomorphism $U$ is a metric automorphism of $\mathscr{C}$.}

Proof. It follows immediately from the definition that $U$ is a $\sigma$-isomorphism of $\mathscr{C}$ onto $\mathscr{C}$. To see that $U$ is $\lambda$-measure preserving, let $E \in \mathscr{C}$ and denote the sequence $\left\{\mu\left(E_{j}\right): j \in Z\right\}$ by $x$, then $T_{1} x=\left\{\mu\left(E_{j+1}\right): j \in Z\right\}=\left\{\mu\left(\left(U^{-1} E\right)_{j}\right): j \in Z\right\}$, and it follows that $\lambda(E)=L(x)=L\left(T_{1} x\right)=\lambda\left(U^{-1} E\right)$.

3.4. THEOREM. For each metric automorphism $T$ of $\mathscr{M}$ there exists a $U$-invariant $\sigma$-subalgebra $\mathscr{A}(T)$ of $\mathscr{C}$ such that $\lambda$ restricted to $\mathscr{A}(T)$ is a countably additive measure, the measure space $(Z \times X, \mathscr{A}(T), \lambda)$ is metrically isomorphic to $(X, \mathscr{M}, \mu)$, and $U$ restricted to $\mathscr{A}(T)$ is conjugate to $T$. Furthermore the algebra $\mathscr{A}(T)$ satisfies the conditions

(i) for every $j \in Z, A \in \mathscr{A}(T), \mu\left(A_{j}\right)=\lambda(A)$,

(ii) for every $E \in \mathscr{M}$, there exists a set $A \in \mathscr{A}(T)$ such that $A_{0}=E$ a.e. $(\mu)$.

Proof. Define the function $\alpha_{T}$ on $\mathscr{M}$ by

$$
\alpha_{T}(E)=\left\{(j, x): x \in T^{j} E\right\}=\bigcup_{j \in Z}\left(\{j\} \times T^{j} E\right) .
$$

Since $T$ is a metric automorphism, $T^{j} E \in \mathscr{M}$ for each $j$ and hence $\alpha_{T}(E) \in \mathscr{C}$. It is easy to see that $\alpha_{T}$ is a $\sigma$-homomorphism of $\mathscr{M}$ into $\mathscr{C}$ and that if we denote the range of $\alpha_{T}$ by $\mathscr{A}(T)$, then $\mathscr{A}(T)$ is a subalgebra of $\mathscr{C}$.

Let $E \in \mathscr{M}$. Since $T$ is measure preserving, $\mu\left(T^{j} E\right)=\mu(E)$ for all $j$ and hence $\mu\left(\alpha_{T}(E)_{j}\right)=\mu(E)$ for all $j$. Thus $\lambda\left(\alpha_{T}(E)\right)=L\left\{\mu\left(\left(\alpha_{T} E\right)_{j}\right): j \in Z\right\}=\mu(E)$ and $\alpha_{T}$ is measure preserving on $(X, \mathscr{M}, \mu)$ onto $(Z \times X, \mathscr{A}(T), \lambda)$.

Suppose $\alpha_{T}(E)=\alpha_{T}(F)$ a.e. Then if $\triangle$ denotes symmetric difference $\alpha_{T}(E \triangle F)=\varnothing$, a.e., and $0=\lambda\left(\alpha_{T}(E \triangle F)\right)=\mu(E \triangle F)$ so that $E=F$, a.e. Thus $\alpha_{T}$ is one-to-one on the measure algebras, and $\alpha_{T}$ is a metric $\sigma$-isomorphism on $(X, \mathscr{M}, \mu)$ onto $(Z \times X, \mathscr{A}(T), \lambda)$, and since $\mu$ is countably additive, $\lambda$ is countably additive on $\mathscr{A}(T)$.

To show that $T$ is conjugate to $U$ restricted to $\mathscr{A}(T)$, let $E \in \mathscr{M}$. Then

$$
\begin{aligned}
U \circ \alpha_{T}(E) & =U\left(\bigcup_{j \in Z}\left(\{j\} \times T^{j} E\right)\right) \\
& =\bigcup_{j \in Z}\left(\{j-1\} \times T^{j} E\right) \\
& =\bigcup_{j \in Z}\left(\{j\} \times T^{j}(T E)\right) \\
& =\alpha_{T} \circ T(E) .
\end{aligned}
$$

This also shows that $\mathscr{A}(T)$ is $U$-invariant. 
Finally, to see that (i) and (ii) are satisfied, by the definition of $\mathscr{A}(T)$, given $A \in \mathscr{A}(T)$, there is $E \in \mathscr{M}$ such that $\alpha_{T}(E)=A$ and $\lambda(A)=\mu(E)=\mu\left(T^{j} E\right)=\mu\left(A_{j}\right)$ for each $j \in Z$ so that (i) is satisfied. Also given $E \in \mathscr{M},\left(\alpha_{T}(E)\right)_{0}=E$ and condition (ii) is satisfied.

3.5. TheOREM. Let $\mathscr{A}$ be a U-invariant subalgebra of $\mathscr{C}$ such that conditions (i) and (ii) of Theorem 3.4 are satisfied. Then there exists a metric automorphism $T$ of $\mathscr{M}$ such that $U$ restricted to $\mathscr{A}$ is conjugate to $T$.

Proof. For $E \in \mathscr{A}$ let $\beta(E)=E_{0}=\{x:(0, x) \in E\}$. Then $\beta$ is a $\sigma$-homomorphism of $\mathscr{A}$ into $\mathscr{M}$ and it follows from 3.4 (ii) that $\beta$ is onto and from 3.4 (i) that $\mu(\beta(E))=\lambda(E)$ so that $\beta$ is a metric $\sigma$-homomorphism of $\mathscr{A}$ onto $\mathscr{M}$. Suppose $\beta\left(E_{1}\right)=\beta\left(E_{2}\right)$ a.e. Then $\beta\left(E_{1} \triangle E_{2}\right)=\varnothing$ a.e. and $\lambda\left(E_{1} \triangle E_{2}\right)=\mu\left(\beta\left(E_{1} \triangle E_{2}\right)\right)=0$ so that $E_{1}=E_{2}$ a.e. and $\beta$ is one-to-one. Now define $T$ on $\mathscr{M}$ by $T=\beta U \beta^{-1}$ and $T$ is conjugate to $U$ restricted to $\mathscr{A}$.

4. Applications. For each $T \in \mathscr{G}$, denote by $\mathscr{A}(T)$ the algebra given by Theorem 3.4 and by $\alpha_{T}$ and $\beta$ the like named metric $\sigma$-isomorphisms defined in Theorems 3.4 and 3.5. Note that $\alpha_{T}$ maps $\mathscr{M}$ onto $\mathscr{A}(T)$ and $\beta$, being the projection onto the 0th component maps $\mathscr{C}$ onto $\mathscr{M}$. If we denote the restriction of $\beta$ to $\mathscr{A}(T)$ by $\beta_{T}$, then $\beta_{T}$ maps $\mathscr{A}(T)$ onto $\mathscr{M}$ and $\alpha_{T}^{-1}=\beta_{T}$. Let $I$ denote the identity automorphism in $\mathscr{G}$, and 2 denote the trivial subalgebra consisting of $\varnothing$ and the whole space.

\subsection{THEOREM. The automorphism $T$ is ergodic iff $\mathscr{A}(T) \cap \mathscr{A}(I)=2$.}

Proof. Suppose $T$ is ergodic. Then given $E \in \mathscr{M}, T E=E$ implies that $E=\varnothing$ or $X$. Let $F \in \mathscr{A}(T) \cap \mathscr{A}(I)$. Then $\bigcup_{j \in Z}\left(\{j\} \times T^{j} F_{0}\right)=\bigcup_{j \in Z}\left(\{j\} \times F_{0}\right)$ and hence $T^{j} F_{0}=F_{0}$ for all $j$ and $F_{0}$ must be either $\varnothing$ or $X$ and $F$ is either $\varnothing$ or $Z \times X$. Thus $\mathscr{A}(T) \cap \mathscr{A}(I) \subset 2$. Since the reverse inclusion is always satisfied, $\mathscr{A}(T) \cap \mathscr{A}(I)=2$.

Suppose $\mathscr{A}(T) \cap \mathscr{A}(I)=2$. Then for any set $E \in \mathscr{M}$ such that $T E=E, T^{j} E=E$ for all $j$ and it follows that $\bigcup_{j \in Z}\left(\{j\} \times T^{j} E\right)=\bigcup_{j \in Z}(\{j\} \times E)=F \in \mathscr{A}(T) \cap \mathscr{A}(I)$. Thus $F$ is either $\varnothing$ or $Z \times X$. In the first case $E=\varnothing$ and in the second $E=X$.

4.2. THEOREM. If $T$ is ergodic then $\mathscr{A}(T)$ and $\mathscr{A}(I)$ are $\lambda$-independent.

Proof. Since $T$ is ergodic, for sets $E, F \in \mathscr{M}$,

$$
\lim _{n} \frac{1}{n} \sum_{j=0}^{n-1} \mu\left(T^{j} E \cap F\right)=\mu(E) \mu(F) .
$$

For a proof of this statement see [3, p. 33]. 
Let $A \in \mathscr{A}(T)$ and $Z \times F \in \mathscr{A}(I)$. Then $F \in \mathscr{M}$ and

$$
\begin{aligned}
\lambda(A \cap(Z \times F)) & =L\left\{\mu\left(A_{j} \cap F\right): j \in Z\right\} \\
& =\lim _{n} \frac{1}{n} \sum_{j=0}^{n-1} \mu\left(T^{j} A_{0} \cap F\right) \\
& =\mu\left(A_{0}\right) \mu(F)=\lambda(A) \lambda(Z \times F) .
\end{aligned}
$$

4.3. Lemma. Let $T, S \in \mathscr{G} ; \mathscr{M}^{\prime}=\beta(\mathscr{A}(T) \cap \mathscr{A}(S))$. Then $\mathscr{M}^{\prime}$ is the maximal subalgebra of $\mathscr{M}$ which is both $T$ and $S$ invariant and such that $T$ and $S$ coincide on $\mathscr{M}^{\prime}$. Furthermore, if $T$ is ergodic, every $T$ invariant subalgebra of $\mathscr{M}$ may be represented by $\beta(\mathscr{A}(T) \cap \mathscr{A}(R))$ for some $R$ in $\mathscr{G}$.

Proof. Suppose that $\mathscr{M}^{*}$ is a subalgebra of $\mathscr{M}$ which is both $T$ and $S$ invariant and such that $T=S$ on $\mathscr{M}^{*}$. Let $E \in \mathscr{M}^{*}$. Then $T^{j} E=S^{j} E$ for all $j \in Z$ and hence $\alpha_{T}(E)=\alpha_{S}(E)$ and $\mathscr{M}^{*} \subset \beta(\mathscr{A}(T) \cap \mathscr{A}(S))=\mathscr{M}^{\prime}$. It follows easily from the definition of $\mathscr{M}^{\prime}$ that it is both $T$ and $S$ invariant and that $T=S$ on $\mathscr{M}^{\prime}$.

Suppose $T$ is ergodic. Let $\mathscr{M}^{*}$ be a $T$-invariant subalgebra of $\mathscr{M}$. For each $E \in \mathscr{M}^{*}$ define $R E$ to be $T E$ and for $E \in \mathscr{M}-\mathscr{M}^{*}$ define $R E$ to be $E$. Then $R$ is a metric automorphism and since $T$ is ergodic $\mathscr{M}^{*}$ is the maximal subalgebra on which $T$ and $R$ coincide. Thus $\mathscr{M}^{*}=\beta(\mathscr{A}(T) \cap \mathscr{A}(R))$.

For $T \in \mathscr{G}$ and $\mathscr{B}$ a $T$-invariant subalgebra of $\mathscr{M}$ denote the restriction of $T$ to $\mathscr{B}$ by $T \mid \mathscr{B}$. An automorphism has completely positive entropy (see [6]) if for each nontrivial $T$ invariant subalgebra $\mathscr{B}$ of $\mathscr{M}, h(T \mid \mathscr{B})>0$. If $\mathscr{B}_{1} \subset \mathscr{B}_{2}$, then $h\left(T \mid \mathscr{B}_{1}\right)<h\left(T \mid \mathscr{B}_{2}\right)$ (see [7]).

4.4. Lemma. If $T, S \in \mathscr{G}, T$ has completely positive entropy, and $\mathscr{A}(T) \cap \mathscr{A}(S) \neq 2$, then $S$ has positive entropy.

Proof. Since $\mathscr{A}(T) \cap \mathscr{A}(S) \neq 2, \beta(\mathscr{A}(T) \cap \mathscr{A}(S)) \neq 2$. Thus

$$
0<h(T \mid \beta(\mathscr{A}(T) \cap \mathscr{A}(S)))=h(S \mid \beta(\mathscr{A}(T) \cap \mathscr{A}(S))) \leqq h(S) .
$$

\subsection{THEOREM( $\left.{ }^{2}\right)$. If $T$ has completely positive entropy then $T$ is ergodic.}

Proof. By Theorem 4.1 if $T$ is not ergodic, $\mathscr{A}(T) \cap \mathscr{A}(I) \neq 2$. This implies by Lemma 4.4 that $I$ has positive entropy which is false.

(2) A much stronger result than this is stated without proof by Rohlin [7, p. 14] namely that automorphisms with completely positive entropy are mixing of all orders. 
4.6. LEMMA. If $T \in \mathscr{G}$ and $F$ is a function on $Z \times X$ to the reals which is $\mathscr{A}(T)$ measurable and $\lambda$-summable, then $F(0, \cdot)$ is $\mu$-summable and

$$
\int_{Z \times X} F d \lambda=\int_{X} F(0, \cdot) d \mu
$$

Proof. Suppose $B$ is a real Borel set. Then since $F$ is $\mathscr{A}(T)$-measurable $F^{-1}(B) \in$ $\mathscr{A}(T)$ or

$$
\{(n, x): F(n, x) \in B\}=\bigcup_{n \in Z}\left(\{n\} \times T^{n}\{x: F(0, x) \in B\}\right)
$$

so that $\{x: F(0, x) \in B\}$ is in $\mathscr{M}$ and $F(0, \cdot)$ is $\mathscr{M}$-measurable.

Since $F$ is $\lambda$-summable, there exists a sequence $\left\{F_{j}\right\}$ of simple functions which converges a.e. to $F_{0}$. Suppose $F_{j}(n, x)=\sum_{k} C_{k}^{j} \chi_{E_{k j}}(n, x)$. Then

$$
\begin{aligned}
\int_{z \times X} F_{j} d \lambda & =\sum_{k} C_{k}^{j} \lambda\left(E_{k j}\right)=\sum_{k} C_{k}^{j} \mu\left\{\left(E_{k j}\right)_{0}\right\} \\
& =\sum_{k} C_{k}^{j} \int_{X} \chi_{E_{k j}}(0, \cdot) d \mu \\
& =\int_{X} F_{j}(0, \cdot) d \mu .
\end{aligned}
$$

Since $F_{j}(0, x)$ converges a.e. to $F(0, x)$, we have that $\int_{Z \times X} F d \lambda=\int_{X} F(0, \cdot) d \mu$. Let $\xi=\left\{A_{1}, \ldots, A_{k}\right\}$ be a finite measurable partition of $X$. For $T \in \mathscr{G}$ denote the partition $\left\{\alpha_{T} A_{j}: j=1, \ldots, k\right\}$ of $Z \times X$ by $\alpha_{T} \xi$, and if $\mathscr{B}$ is a subalgebra of $\mathscr{C}$ denote the subalgebra $\{\beta(E): E \in \mathscr{B}\}$ of $\mathscr{M}$ by $\beta(\mathscr{B})$. If $F$ is a function on $Z \times X$, $F_{0}$ will denote the function $F(0, \cdot)$. If $h$ is a $\mu$-summable function and $\mathscr{A}$ is a subalgebra of $\mathscr{M}$, denote by $E_{\mu}(h \mid \mathscr{A})$ the conditional expectation of $h$ given $\mathscr{A}$ and if $f$ is an $\mathscr{A}(T)$-measurable, $\lambda$-summable function and $\mathscr{B}$ is a subalgebra of $\mathscr{A}(T)$ let $E_{\lambda}(f \mid \mathscr{B})$ denote the conditional expectation of $f$ given $\mathscr{B}$. For properties of conditional expectation see [5].

4.7. LeMmA. If $T, S \in \mathscr{G}, f$ is $\mathscr{A}(T)$-measurable and $\lambda$-summable then

$$
\left[E_{\lambda}(f \mid \mathscr{A}(T) \cap \mathscr{A}(S))\right]_{0}=E_{\mu}\left(f_{0} \mid \beta(\mathscr{A}(T) \cap \mathscr{A}(S))\right) \text { a.e. }
$$

Proof. By Lemma 4.6, $f_{0}$ is $\mu$-summable and $\int f d \lambda=\int f_{0} d \mu$. By the definition of $E_{\lambda}(f \mid \mathscr{A}(T) \cap \mathscr{A}(S))$, this function is $\mathscr{A}(T) \cap \mathscr{A}(S)$-measurable and for any $E \in \mathscr{A}(T) \cap \mathscr{A}(S)$

$$
\int_{E} E_{\lambda}(f \mid \mathscr{A}(T) \cap \mathscr{A}(S)) d \lambda=\int_{B(E)} f_{0} d \mu .
$$


Since $f_{0}$ is $\mu$-summable, by definition of conditional expectation,

$$
E_{\mu}\left(f_{0} \mid \beta(\mathscr{A}(T) \cap \mathscr{A}(S))\right)
$$

is $\beta(\mathscr{A}(T) \cap \mathscr{A}(S))$-measurable and for each $\beta(E) \in \beta(\mathscr{A}(T) \cap \mathscr{A}(S))$,

$$
\int_{\beta(E)} E_{\mu}\left(f_{0} \mid \beta(\mathscr{A}(T) \cap \mathscr{A}(S))\right) d \mu=\int_{\beta(E)} f_{0} d \mu .
$$

Thus for each $E \in(\mathscr{A}(T) \cap \mathscr{A}(S))$,

$$
\int_{E} E_{\lambda}(f \mid \mathscr{A}(T) \cap \mathscr{A}(S)) d \lambda=\int_{\beta(E)} E_{\mu}\left(f_{0} \mid \beta(\mathscr{A}(T) \cap \mathscr{A}(S))\right) d \mu .
$$

However, from Lemma 4.6,

$$
\int \chi_{E} E_{\lambda}(f \mid \mathscr{A}(T) \cap \mathscr{A}(S)) d \lambda=\int \chi_{\beta(E)}\left[E_{\lambda}(f \mid \mathscr{A}(T) \cap \mathscr{A}(S))\right]_{0} d \mu
$$

for every set $E \in \mathscr{A}(T) \cap \mathscr{A}(S)$. Thus for every set $E \in \mathscr{A}(T) \cap \mathscr{A}(S)$,

$$
\int_{\beta(E)}\left[E_{\lambda}(f \mid \mathscr{A}(T) \cap \mathscr{A}(S))\right]_{0} d \mu=\int_{B(E)} E_{\mu}\left(f_{0} \mid \beta(\mathscr{A}(T) \cap \mathscr{A}(S))\right) d \mu .
$$

Moreover, since $E_{\lambda}(f \mid \mathscr{A}(T) \cap \mathscr{A}(S))$ is $\mathscr{A}(T) \cap \mathscr{A}(S)$-measurable,

$$
\left[E_{\lambda}(f \mid \mathscr{A}(T) \cap \mathscr{A}(S))\right]_{0}
$$

is $\beta(\mathscr{A}(T) \cap \mathscr{A}(S))$-measurable and it follows that

$$
\left[E_{\lambda}(f \mid \mathscr{A}(T) \cap \mathscr{A}(S))\right]_{0}=E_{\mu}\left(f_{0} \mid \beta(\mathscr{A}(T) \cap \mathscr{A}(S))\right) \text { a.e. }
$$

4.8. Corollary. If $A \in \mathscr{M}$ and $T, S \in \mathscr{G}$, then

$$
\left[\lambda\left(\alpha_{T} A \mid \mathscr{A}(T) \cap \mathscr{A}(S)\right)\right]_{0}=\mu(A \mid \beta(\mathscr{A}(T) \cap \mathscr{A}(S))) .
$$

4.9. LeMMA. Let $\xi$ be any finite measurable partition of $X$ and $T, S \in \mathscr{G}$. Then

$$
\bar{H}\left(\alpha_{T} \xi \mid \mathscr{A}(T) \cap \mathscr{A}(S)\right)=\bar{H}(\xi \mid \beta(\mathscr{A}(T) \cap \mathscr{A}(S))) .
$$

Proof. Suppose $\xi=\left\{A_{1}, \ldots, A_{k}\right\}$ and denote $\alpha_{T} A_{j}$ by $A_{j}^{\prime}$ and $\mathscr{A}(T) \cap \mathscr{A}(S)$ by $\mathscr{B}$. Then by definition of relative entropy and Corollary 4.8

$$
\begin{aligned}
{\left[H\left(\alpha_{T} \xi \mid \mathscr{B}\right)\right]_{0} } & =-\sum_{j=1}^{k}\left[\lambda\left(A_{j}^{\prime} \mid \mathscr{B}\right)\right]_{0} \log \left[\lambda\left(A_{j}^{\prime} \mid \mathscr{B}\right)\right]_{0} \\
& =-\sum_{j=1}^{k} \mu\left(A_{j} \mid \beta(\mathscr{B})\right) \log \mu\left(A_{j} \mid \beta(\mathscr{B})\right) \\
& =H(\xi \mid \beta(\mathscr{B})) \quad \text { a.e. }
\end{aligned}
$$


Thus by Lemma 4.6

$$
\begin{aligned}
\bar{H}\left(\alpha_{T} \xi \mid \mathscr{B}\right) & =\int_{Z \times X} H\left(\alpha_{T} \xi \mid \mathscr{B}\right) d \lambda=\int_{X}\left[H\left(\alpha_{T} \xi \mid \mathscr{B}\right)\right]_{0} d \mu \\
& =\int_{X} H(\xi \mid \beta(\mathscr{B})) d \mu=\bar{H}(\xi \mid \beta(\mathscr{B})) .
\end{aligned}
$$

4.10. Lemma. Let $(Y, \mathscr{N}, v)$ be a probability space and $\mathscr{B}$ a subalgebra of $\mathscr{N}$. A necessary and sufficient condition that $\mathscr{B}=\mathscr{N}$ (a.e.) is that for every finite measurable partition $\xi$ of $Y, \bar{H}(\xi \mid \mathscr{B})=0$.

Proof. It is known [2, p. 31] that $\bar{H}(\xi \mid \mathscr{B})=0$ iff $\xi$ is a finite partition of $Y$ contained a.e. in $\mathscr{B}$, i.e., for each $A \in \xi$, there exists $B \in \mathscr{B}$ such that $A=B$ a.e. Suppose $\mathscr{B}$ does not equal $\mathscr{N}$ a.e. Then there is a set $E \in \mathscr{N}$ such that $\nu(E \triangle B)>0$ for every set $B \in \mathscr{B}$. Consider the partition $\zeta=\{E, Y-E\}$. Then $\bar{H}(\zeta \mid \mathscr{B}) \neq 0$, since if it were zero the partition $\zeta$ would be contained a.e. in $\mathscr{B}$ and there would exist $B \in \mathscr{B}$ such that $\nu(E \triangle B)=0$. In the case $\mathscr{B}=\mathscr{N}$ a.e., every partition of $Y$ is contained a.e. in $\mathscr{B}$ and $\bar{H}(\xi \mid \mathscr{B})=0$.

4.11. Definition. For $T, S \in \mathscr{G}$ define $h(T, S)$ by the equation $h(T, S)=\inf \left\{\sup \bar{H}\left(\alpha_{T} \xi \mid \mathscr{A}(T) \cap \mathscr{A}\left(R^{-1} S R\right)\right): \xi\right.$ finite partition of $\left.X: R \in \mathscr{G}\right\}$.

4.12. THEOREM. Let $T, S \in \mathscr{G}$. If $T$ and $S$ are conjugate then $h(T, S)=0$.

Proof. Since $T$ is conjugate to $S$ there exists $R \in \mathscr{G}$ such that $T=R^{-1} S R$ and by Lemma $4.3 \beta\left[\mathscr{A}(T) \cap \mathscr{A}\left(R^{-1} S R\right)\right]=\mathscr{M}$. Hence by Lemmas 4.10 and 4.9, for each finite partition $\xi$ of $X$,

$$
\begin{aligned}
0 & =\bar{H}\left(\xi \mid \beta\left(\mathscr{A}(T) \cap \mathscr{A}\left(R^{-1} S R\right)\right)\right) \\
& =\bar{H}\left(\alpha_{T} \xi \mid \mathscr{A}(T) \cap \mathscr{A}\left(R^{-1} S R\right)\right) .
\end{aligned}
$$

Thus $h(T, S)=0$.

4.13. Lemma. If $R, S, T \in \mathscr{G}$, then

$$
R[\beta(\mathscr{A}(T) \cap \mathscr{A}(S))]=\beta\left(\mathscr{A}\left(R T R^{-1}\right) \cap \mathscr{A}\left(R S R^{-1}\right)\right) .
$$

Proof. Let $E \in \beta(\mathscr{A}(T) \cap \mathscr{A}(S))$. Then $T^{j} E=S^{j} E$ for all $j \in Z$ since $\alpha_{T}(E)=$ $\alpha_{S}(E)$. Let $F=R E$. Then $R T^{j} R^{-1} F=R S^{j} R^{-1} F$ for all $j$. Since $\left(R T R^{-1}\right)^{j} F=$ $R T^{j} R^{-1} F, \alpha_{R T R^{-1}}(F)=\alpha_{R S R^{-1}}(F)$ and $F=R E \in \beta\left(\mathscr{A}\left(R T R^{-1}\right) \cap \mathscr{A}\left(R S R^{-1}\right)\right)$. Thus $R \beta(\mathscr{A}(T) \cap \mathscr{A}(S)) \subset \beta\left(\mathscr{A}\left(R T R^{-1}\right) \cap \mathscr{A}\left(R S R^{-1}\right)\right)$. Next suppose that

$$
F \in \beta\left(\mathscr{A}\left(R T R^{-1}\right) \cap \mathscr{A}\left(R S R^{-1}\right)\right) .
$$

Then by the first part of the proof $R^{-1} F \in \beta(\mathscr{A}(T) \cap \mathscr{A}(S))$ and the result follows. 
4.14. THEOREM. If $T, S \in \mathscr{G}$, then $h(T, S)=h(S, T)$.

Proof. It is true that if $R \in \mathscr{G}, \bar{H}(R \xi \mid R \mathscr{A})=\bar{H}(\xi \mid \mathscr{A})$. (See [2, p. 31].) Thus, for any finite partition $\xi$ of $X$, any $R \in \mathscr{G}$,

$$
\begin{aligned}
\bar{H}\left(\alpha_{S} \xi \mid \mathscr{A}(S) \cap \mathscr{A}\left(R^{-1} T R\right)\right) & =\bar{H}\left(\xi \mid \beta\left(\mathscr{A}(S) \cap \mathscr{A}\left(R^{-1} T R\right)\right)\right) \\
& =\bar{H}\left(R \xi \mid R \beta\left(\mathscr{A}(S) \cap \mathscr{A}\left(R^{-1} T R\right)\right)\right) \\
& =\bar{H}\left(\alpha_{T}(R \xi) \mid \mathscr{A}\left(R S R^{-1}\right) \cap \mathscr{A}(T)\right) .
\end{aligned}
$$

Thus

$$
\sup _{\xi} \bar{H}\left(\alpha_{S} \xi \mid \mathscr{A}(S) \cap \mathscr{A}\left(R^{-1} T R\right)\right)=\sup _{\xi^{\prime}} \bar{H}\left(\alpha_{T} \xi^{\prime} \mid \mathscr{A}\left(R S R^{-1}\right) \cap \mathscr{A}(T)\right)
$$

and

$\inf _{R \in \mathscr{G}} \sup _{\xi} \bar{H}\left(\alpha_{S} \xi \mid \mathscr{A}(S) \cap \mathscr{A}\left(R^{-1} T R\right)\right)=\inf _{R^{\prime} \in \mathscr{S}} \sup _{\xi^{\prime}} \bar{H}\left(\alpha_{T} \xi^{\prime} \mid \mathscr{A}\left(R^{\prime-1} S R^{\prime}\right) \cap \mathscr{A}(T)\right)$.

4.15. TheOREM. Let $T, S, U \in \mathscr{G}$. Then

$$
h\left(T, U^{-1} S U\right)=h(T, S) .
$$

Proof.

$$
h\left(T, U^{-1} S U\right)=\inf _{R} \sup _{\xi} \bar{H}\left(\alpha_{T} \xi \mid \mathscr{A}(T) \cap \mathscr{A}\left(R^{-1} U^{-1} S U R\right)\right) .
$$

Define $R_{1}=U R$ so that $R=U^{-1} R_{1}$ and

$$
\begin{aligned}
h\left(T, U^{-1} S U\right) & =\inf _{U^{-1}{ }_{R}} \sup _{\xi} \bar{H}\left(\alpha_{T} \xi \mid \mathscr{A}(T) \cap \mathscr{A}\left(R_{1}^{-1} S R_{1}\right)\right) \\
& =h(T, S) .
\end{aligned}
$$

4.16. Corollary. If $T, S, U_{1}, U_{2}$ are contained in $\mathscr{G}$ then

$$
h\left(U_{1}^{-1} T U_{1}, U_{2}^{-1} S U_{2}\right)=h(T, S) .
$$

Proof.

$$
\begin{aligned}
h\left(U_{1}^{-1} T U_{1}, U_{2}^{-1} S U_{2}\right) & =h\left(U_{1}^{-1} T U_{1}, S\right) \\
& =h\left(S, U_{1}^{-1} T U_{1}\right) \\
& =h(S, T)=h(T, S)
\end{aligned}
$$

4.17. THEOREM. If $T \in \mathscr{G}$ and $h(T, I)=0$, then $T=I$. 
Proof.

$$
\begin{aligned}
0=h(T, I) & =\inf _{R \in \mathscr{G}} \sup _{\xi} \bar{H}\left(\alpha_{T} \xi \mid \mathscr{A}(T) \cap \mathscr{A}\left(R^{-1} I R\right)\right) \\
& =\sup _{\xi} \bar{H}\left(\alpha_{T} \xi \mid \mathscr{A}(T) \cap \mathscr{A}(I)\right) .
\end{aligned}
$$

Thus $\bar{H}(\xi \mid \beta(\mathscr{A}(T) \cap \mathscr{A}(I)))=0$ for every partition $\xi$ of $X$ and by Lemma 4.10, $\beta(\mathscr{A}(T) \cap \mathscr{A}(I))=\mathscr{M}$. Thus by Lemma $4.3 T=I$ on $\mathscr{M}$.

4.18. THEOREM. Let $T, S \in \mathscr{G}$. If $h(T, S)=0$ then there exists a sequence $\left\{R_{n}\right\}$ of elements from $\mathscr{G}$ and a sequence $\left\{\mathscr{B}_{n}\right\}$ of $T$-invariant subalgebras of $\mathscr{M}$ such that $T=R_{n}^{-1} S R_{n}$ on $\mathscr{B}_{n}$ and $\bigvee_{n=1}^{\infty} \mathscr{B}_{n}=\mathscr{M}$.

Proof. For each positive integer $n$ there exists an automorphism $R_{n}$ such that $\sup _{\xi} \bar{H}\left(\alpha_{T} \xi \mid \mathscr{A}(T) \cap \mathscr{A}\left(R_{n}^{-1} S R_{n}\right)\right)<1 / n$ and it follows that

$$
\bar{H}\left(\alpha_{T} \xi \mid \mathscr{A}(T) \cap \mathscr{A}\left(R_{n}^{-1} S R_{n}\right)\right)<1 / n
$$

for every finite partition of $X$. If $\mathscr{B}_{n}=\beta\left(\mathscr{A}(T) \cap \mathscr{A}\left(R_{n}^{-1} S R_{n}\right)\right)$. Then $T=R_{n}^{-1} S R_{n}$ on $\mathscr{B}_{n}$ and $\lim _{n \rightarrow \infty} \bar{H}\left(\xi \mid \mathscr{B}_{n}\right)=0$ uniformly in $\xi$. Since

$$
\bigvee_{j=1}^{n} \mathscr{B}_{j} \supset \mathscr{B}_{n}, 0 \leqq \bar{H}\left(\xi \mid \bigvee_{j=1}^{n} \mathscr{B}_{j}\right) \leqq \bar{H}\left(\xi \mid \mathscr{B}_{n}\right)
$$

and $\lim _{n \rightarrow \infty} \bar{H}\left(\xi \mid \bigvee_{j=1}^{n} \mathscr{B}_{j}\right)=0$. Since $\bigvee_{j=1}^{n} \mathscr{B}_{j}$ is an increasing sequence of subalgebras,

$$
\lim _{n \rightarrow \infty} \bar{H}\left(\xi \mid \bigvee_{j=1}^{n} \mathscr{B}_{j}\right)=\bar{H}\left(\xi \mid \bigvee_{j=1}^{\infty} \mathscr{B}_{j}\right) \text { for all } \xi
$$

and it follows from Lemma 4.10 that $\bigvee_{j=1}^{\infty} \mathscr{B}_{j}=\mathscr{M}$.

4.19. THEOREM. If $T, S \in \mathscr{G}$ and $h(T, S)=0$ then for every $\varepsilon>0$, there exists an $R \in \mathscr{G}$ and a T-invariant subalgebra $\mathscr{B}$ of $\mathscr{M}$ such that $T=R^{-1} S R$ on $\mathscr{B}$ and for each measurable set $A$ not in $\mathscr{B}, \mu\left(T A \triangle R^{-1} S R A\right)<\varepsilon$.

Proof. Let $\varepsilon>0$ be given. Since $h(T, S)=0$, there exists an automorphism $R$ such that $\sup _{\xi} \bar{H}\left(\alpha_{T} \xi \mid \mathscr{A}(T) \cap \mathscr{A}\left(R^{-1} S R\right)\right)<\varepsilon^{2} / 16$. Let $\mathscr{B}$ be the algebra $\beta\left(\mathscr{A}(T) \cap \mathscr{A}\left(R^{-1} S R\right)\right)$. Then $\mathscr{B}$ is $T$-invariant and $T=R^{-1} S R$ on $\mathscr{B}$.

Suppose $A \in \mathscr{M}-\mathscr{B}$. Then if $\xi$ is the partition $\{A, X-A\}$, it follows that

$$
\begin{aligned}
0<\bar{H}(\xi \mid \mathscr{B}) & =-\int_{A} \log \mu(A \mid \mathscr{B}) d \mu-\int_{X-A} \log \mu(X-A \mid \mathscr{B}) d \mu \\
& <\varepsilon^{2} / 16
\end{aligned}
$$


and hence $-\int_{A} \log \mu(A \mid \mathscr{B}) d \mu<\varepsilon^{2} / 16$. Since this integral exists, $-\log \mu(A \mid \mathscr{B})<\infty$ a.e. on $A$ and hence $\mu(A \mid \mathscr{B})>0$ a.e. on $A$. Thus $-\log \mu(A \mid \mathscr{B})>1-\mu(A \mid \mathscr{B})$ a.e. on $A$ and it follows that

$$
\int_{A}[1-\mu(A \mid \mathscr{B})] d \mu<\frac{\varepsilon^{2}}{16}
$$

Now let $B=\{x: \mu(A \mid \mathscr{B})(x)>1-\varepsilon / 4\}$. Since $B \in \mathscr{B}$ we have

$$
\mu(A \cap B)=\int_{B} \mu(A \mid \mathscr{B}) d \mu>(1-\varepsilon / 4) \mu(B)
$$

and it follows that $\mu(B-A)<\varepsilon / 4$. Also

$$
\begin{aligned}
\frac{\varepsilon^{2}}{16}>\int_{A}[1-\mu(A \mid \mathscr{B})] d \mu & =\int_{A-B}[1-\mu(A \mid \mathscr{B})] d \mu+\int_{A \cap B}[1-\mu(A \mid \mathscr{B})] d \mu \\
& \geqq \frac{\varepsilon}{4} \cdot \mu(A-B)
\end{aligned}
$$

so that $\mu(A-B)<\varepsilon / 4$. Thus $\mu(A \triangle B)<\varepsilon / 2$. Since $B \in \mathscr{B}$ and $T=R^{-1} S R$ on $\mathscr{B}$,

$$
\begin{aligned}
\mu\left(T A \triangle R^{-1} S R A\right) & \leqq \mu(T A \triangle T B)+\mu\left(T B \triangle R^{-1} S R B\right)+\mu\left(R^{-1} S R B \triangle R^{-1} S R A\right) \\
& =\mu(A \triangle B)+\mu(B \triangle A) \\
& <\varepsilon .
\end{aligned}
$$

4.20. Corollary. If $h(T, S)=0$ and if the conjugacy class of $S$ is closed in the uniform topology on $\mathscr{G}$ then $T$ and $S$ are conjugate.

Proof. Let $\varepsilon>0$ be given. Recall that $\mathscr{G}$ with the uniform topology (see [3, p. 69]) is a metric group with metric given by

$$
d\left(T_{1}, T_{2}\right)=\sup \left\{\mu\left(T_{1} E \triangle T_{2} E\right): E \in \mathscr{M}\right\} .
$$

Denote by $K$ the conjugacy class of $S$, i.e., the set $\left\{R^{-1} S R: R \in \mathscr{G}\right\}$. Now, if $h(T, S)=0$, it follows from Theorem 4.19 that there exists an $R$ such that $\mu\left(T A \triangle R^{-1} S R A\right)<\varepsilon$ for every set $A \in \mathscr{M}$. Thus $d\left(T, R^{-1} S R\right)<\varepsilon$ and it follows that $T$ is a limit point of $K$. Since $K$ is assumed closed, $T \in K$ and the theorem follows.

\section{BIBLIOGRAPHY}

1. Thomas A. Brown, Entropy and conjugacy, Ann. Math. Statist. 34 (1963), 226-232.

2. Paul R. Halmos, Entropy in ergodic theory, Mimeographed lecture notes, Univ. of Chicago, Chicago, Ill., 1959.

3. - Lectures on ergodic theory, Chelsea, New York, 1956. 
4. K. Jacobs, Lecture notes on ergodic theory, Universitet Aarhus, 1962/1963.

5. Michel Loève, Probability theory, 2nd ed., Van Nostrand, Princeton, N. J., 1960.

6. M. S. Pinsker, Dynamical systems with completely positive or zero entropy, Dokl. Akad. Nauk SSSR 133 (1960), 1025-1026=Soviet Math. Dokl. 1 (1960), 937-938.

7. V. A. Rohlin, New progress in the theory of transformations with invariant measure, Uspehi Math. Nauk 15 (1960), 3-26=Russian Math. Surveys 15 (1960), 1-22.

8. Gian-Carlo Rota, On the classification of periodic flows, Proc. Amer. Math. Soc. 13 (1962), 659-662.

9. A. C. Zaanen, An introduction to the theory of integration, Academic Press, New York, 1958.

UNIVERSITY OF VIRGINIA,

Charlottesville, Virginia 